\title{
Metástasis hepática de origen no colorrectal ni neuroendocrino. Tratamiento quirúrgico*
}

\author{
Drs. RICARDO YÁÑEZ M. ${ }^{1}$, CRISTIAN GAMBOA C. ${ }^{1}$, FERNANDO CROVARI E. ${ }^{1}$, \\ SERGIO GUZMÁN B. ${ }^{1}$, JORGE MARTÍNEZ C. ${ }^{1}$, NICOLÁS JARUFE C. ${ }^{1}$, \\ Als. MAXIMILIANO CURI T. ${ }^{2}$, OSVALDO WEISSE A. ${ }^{2}$, JUAN F. GUERRA C. ${ }^{2}$
}

1 Departamento Cirugía Digestiva, División de Cirugía.

2 Facultad de Medicina, Escuela de Medicina.

Pontificia Universidad Católica de Chile. Santiago, Chile.

\begin{abstract}
Surgical excision of non colorectal liver metastases

Background: The benefits of hepatic resections for colorectal liver metastases are well known. This is not the case for excisions in the treatment of non-colorectal, non neuroendocrine liver metastases. Aim: To assess the results of liver resection in the treatment of patients with non-colorectal, non neuroendocrine liver metastases. Material and Methods: Electronic database analysis of patients with noncolorectal non-endocrine liver metastases undergoing to hepatectomy between 2000-2009. Results: Seventeen patients aged 22 to 78 years, nine women, were operated. The most common primary tumors were stomach, liver, adrenal glands and uterus. In two cases, a synchronic primary tumor and metastasis excision was performed. Anatomic segmentectomy was performed in 10 cases $(58.8 \%)$ and a mayor surgical resection in 7 patients $(41.2 \%)$. In 15 cases $(88.2 \%)$ the tumor margins were negative. Three patients presented postoperative complications and three patients had hepatic tumor recurrence. No patient died in the peri-operative period. One, two and three years survival were 85, 51 and 51\% respectively, after a follow-up ranging from 9 to 56 months. Conclusions: The surgical treatment of patients with non-colorectal non-endocrine liver metastases is safe and beneficial in selected patients, with a low rate of complications and good survival rates.
\end{abstract}

Key words: Liver metastases, excision, non colorectal tumors.

\section{Resumen}

Introducción: Existe un claro beneficio en el tratamiento quirúrgico de las metástasis hepáticas de origen colorrectal y neuroendocrinas; sin embargo, todavía no está bien definida la efectividad de la resección quirúrgica en tumores de origen diferente a los anteriores. El objetivo del presente estudio es dar a conocer los resultados del tratamiento quirúrgico en este grupo de pacientes. Pacientes y Método: Análisis de la base de datos electrónica de los pacientes con metástasis hepáticas de origen no colorrectal ni neuroendocrina que fueron sometidos a resección hepática en nuestro centro entre los años 2000-2009. Resultados: La serie estuvo constituida por 17 pacientes, nueve mujeres, mediana de edad de 51 años (rango, 22-78). Los

\footnotetext{
*Recibido el 25 de Agosto de 2009 y aceptado para publicación el 6 de Octubre de 2009.

Correspondencia: Dr. Ricardo Yáñez M.

Fax: 5623536601

Vicuña Mackenna 625, of 820,Santiago, Chile.

E-mail: ryanezm@uc.cl
} 
principales sitios de origen del tumor primario fueron estómago, hígado, glándulas suprarrenales y útero. En dos casos se realizó cirugía sincrónica del primario y las metástasis; la técnica utilizada fue segmentectomía anatómica en 10 pacientes $(58,8 \%)$ y resección anatómica mayor los siete restantes $(41,2 \%)$. En 15 pacientes $(88,2 \%)$ se logró borde quirúrgico libre de tumor, tres pacientes presentaron complicaciones postoperatorias y en tres hubo recidiva de la lesión hepática. No hubo mortalidad operatoria. Tras un seguimiento de 21 meses (rango, 9-56) la supervivencia al año, a los dos y a los tres años fue de $85 \%, 51 \%$ y $51 \%$ respectivamente. Discusión: El tratamiento quirúrgico de los pacientes con metástasis hepáticas de origen no colorrectal ni neuroendocrino es seguro y parece beneficioso en pacientes seleccionados, con baja tasa de complicaciones y con supervivencia favorable.

Palabras clave: Metástasis hepáticas, hepatectomía, neoplasia hepática.

\section{Introducción}

Las metástasis hepáticas son la principal causa de tumores hepáticos malignos y constituyen la segunda localización más frecuente de las neoplasias malignas $^{1,2}$. Los avances en cirugía hepática y el desarrollo de terapias complementarias han permitido que las metástasis hepáticas puedan ser tratadas con intención curativa ${ }^{3}$.

La resección hepática es segura y efectiva en metástasis hepáticas de origen colorrectal, grupo de pacientes en el cual la sobrevida a cinco años es entre $40 \%$ y $50 \%$ posterior al tratamiento quirúrgico, igualmente es sabido que el tratamiento quirúrgico de las metástasis hepáticas de tumores neuroendocrinos tienen una sobrevida a cinco años cercana al $64 \%{ }^{3-9}$. Sin embargo, en el grupo de pacientes con metástasis hepática de origen no colorrectal y no neuroendocrino aun no está bien definida la eficacia del tratamiento quirúrgico debido a la heterogeneidad de los tumores primarios tanto en el sitio de origen como en su histología ${ }^{10-17}$.

El objetivo de este estudio, es evaluar las indicaciones y resultados de las resecciones hepáticas en pacientes con metástasis hepáticas de origen no colorrectal ni neuroendocrino principalmente en términos de técnica empleada, complicaciones y supervivencia.

\section{Material y Método}

\section{Pacientes}

Se realizó un análisis de la base de datos prospectiva de los pacientes con metástasis hepáticas de origen no colorrectal y no neuroendocrino que fueron sometidos a resección hepática en el Hospital Clínico de la Pontificia Universidad Católica de Chile entre los años 2000 y 2008. Se registraron datos demográficos de cada paciente, características del tumor primario, tiempo entre el diagnóstico y tratamiento del tumor primario, y el diagnóstico y resección de las metástasis hepáticas, características de las metástasis hepáticas, hepatectomía realizada, sangrado intraoperatorio, estado de los márgenes quirúrgicos, estadía hospitalaria, morbilidad y mortalidad. Se logró el seguimiento de todos los pacientes.

Se definió MH metacrónica, al diagnóstico de una metástasis después de los tres meses del tratamiento del tumor primario ${ }^{10,18,19}$. Las complicaciones postoperatorias tempranas fueron definidas como aquellas que se presentaron dentro de los primeros 90 días desde la cirugía ${ }^{20}$.

Los pacientes que fueron sometidos a resección hepática estaban en buenas condiciones generales y no presentaban enfermedad diseminada extrahepática no resecable. Fueron evaluados con Tomografía Axial Computada (TAC) de Tórax, Abdomen y Pelvis, Resonancia Magnética (RM) y Tomografía por Emisión de Positrones (PET) en determinados pacientes. Se consideró un volumen residual $>20 \%$ por volumetría como suficiente para las resecciones extendidas en ausencia de enfermedad hepática y $>40 \%$ en pacientes con daño hepático crónico ${ }^{21,22}$.

\section{Hepatectomía}

Se utilizó la clasificación hepática anatómica de Couinaud y la clasificación de resecciones hepáticas del Consenso de Brisbane ${ }^{23-25}$.

Se definió bisegmentectomía como la resección hepática que incluyó hasta dos segmentos hepáticos de Coinaud y resección hepática mayor cuando incluyó a más de dos segmentos anatómicos. Hepatectomía derecha correspondió a la resección de los segmentos 5, 6, 7 y 8 de Couinaud. Resecciones locales fueron aquellas que no siguieron un plano anatómico o fueron sub-segmentarias. Cualquier extensión que incluya 1 ó más segmentos tanto a derecha como a izquierda de las hepatectomías anteriores, se consideró como hepatectomías extendidas $^{26}$. En todos los casos se realizó ecografía intraoperatoria.

\section{Análisis estadístico}

Los datos estadísticos fueron analizados mediante programa SPSS 17.0, para variables continuas se utilizó t-Student y para variables categóricas fue 
aplicado $\chi^{2}$ donde $p$ value $\leq 0,05$ se considera significativo, la sobrevida fue analizada según KaplanMeier y comparada por análisis de Log Rank.

Patrones de recurrencia y causas de muerte fueron evaluadas en todos los pacientes para determinar sobrevida libre de enfermedad y supervivencia acumulada.

\section{Resultados}

Durante el período analizado, de un total de 77 hepatectomías por metástasis hepáticas, la resección de metástasis de origen no colorrectal no neuroendocrino corresponde al $22 \%$ del total.

La serie está compuesta por 17 pacientes, nueve mujeres. La mediana de edad fue de 51 (22-78) años de edad. Los sitios más frecuentes de tumores primarios están descritos en la Tabla 1.

Al ser analizados según histología del tumor primario, se encontró que lo más frecuente fue el adenocarcinoma ( 8 pacientes, 47\%), siguiendo el sarcoma (6 pacientes, 35,3\%), ameloblastoma maligno, GIST y melanoma ( 1 paciente, $6,7 \%$ respectivamente).

El tumor primario había sido tratado previamente con resección quirúrgica en el $88,2 \%$ de los pacientes y en el $11,7 \%$ se realizó resección sincrónica. Como terapia adyuvante del tumor primario se utilizó quimioterapia preoperatoria y postoperatoria en 9 pacientes y en 11 pacientes respectivamente. Las

Tabla 1. Distribución de los pacientes según origen del tumor primario

\begin{tabular}{lcr}
\hline $\begin{array}{l}\text { Origen del tumor } \\
\text { primario }\end{array}$ & $\begin{array}{c}\mathbf{n}=\mathbf{1 7} \\
\text { pacientes }\end{array}$ & $\mathbf{\%}$ \\
\hline Estómago & 2 & 11,9 \\
$\quad$ Ca gástrico & 1 & 5,8 \\
$\quad$ GIST & 1 & 5,8 \\
Mesenterio & & \\
Hígado & 1 & 5,8 \\
$\quad$ Angiosarcoma & 1 & 5,8 \\
$\quad$ Colangiocarcinoma & 2 & 11,9 \\
Gl. suprarrenales & 2 & 11,9 \\
Útero & 1 & 5,8 \\
Óseo & 1 & 5,8 \\
Pulmón & 1 & 5,8 \\
Pleura & 1 & 5,8 \\
Tejido dentario & 1 & 5,8 \\
Páncreas & 1 & 5,8 \\
Mama & 1 & 5,8 \\
Piel & & \\
\hline
\end{tabular}

metástasis hepáticas fueron sincrónicas, es decir, diagnosticadas dentro de los primeros tres meses de tratamiento del tumor primario, en 5 pacientes y metacrónicas en 12 pacientes. El tiempo promedio desde el tratamiento del primario y el diagnóstico de las metástasis fue de 25 meses (rango, 0-169 meses).

Las metástasis hepáticas fueron múltiples en 53\% de los pacientes. La mediana del diámetro mayor de las metástasis fue de $44 \mathrm{~mm}$ (rango, 7-90 mm) y el volumen de éstas fue de $19 \mathrm{ml}$ (rango, 0,8-1.657 $\mathrm{ml})$.

En cuanto a las hepatectomías, la mediana de tiempo desde el diagnóstico de metástasis hepática hasta la resección hepática fue de tres meses (rango 0-18 meses). En el 100\% de los pacientes fue realizada una resección anatómica. Hepatectomías mayores fueron realizadas en el $41,1 \%$ de los pacientes. La descripción según tipo de hepatectomía realizada se resume en la Tabla 2. Se logró márgenes de resección negativos (R0) en 15 pacientes y positivos microscópicamente (R1) en dos pacientes.

En el período de 90 días comprendidos desde la hepatectomía no se registró mortalidad perioperatoria. Hubo complicaciones tempranas en un paciente y correspondió a una trombosis venosa profunda tratada médicamente. Se presentaron complicaciones tardías en 2 pacientes y correspondieron ambos casos a íleos mecánicos por bridas que debieron ser reintervenidos y evolucionaron favorablemente. La estadía hospitalaria postoperatoria fue de 7 días (rango, 4-17 días).

Luego de un seguimiento de 21 meses (rango, 6-56 meses) la mediana de supervivencia fue de 17 meses, teniendo actualmente diez pacientes vivos. Tal como se muestra en la Figura 1 al analizar mediante Kaplan-Meier se obtiene una supervivencia acumulada de $84,8 \%$ al año de seguimiento, 50,9\% al segundo año y $50,9 \%$ al tercer año de seguimiento.

Al evaluar la supervivencia libre de enfermedad se observó que durante el seguimiento hubo recurrencia de las metástasis en cinco pacientes, dos de

\section{Tabla 2. Tipos de hepatectomías realizadas a los pacientes con metástasis hepática de origen no colorrectal sometidos a resección quirúrgica}

\begin{tabular}{lcr}
\hline Tipo de resección hepática & $\begin{array}{c}\mathbf{n}=\mathbf{1 7} \\
\text { pacientes }\end{array}$ & $\mathbf{\%}$ \\
\hline Segmentectomía & 10 & 58,8 \\
Hepatectomía izquierda & 3 & 17,6 \\
Hepatectomía izquierda extendida & 1 & 5,8 \\
Hepatectomía derecha & 2 & 11,8 \\
Hepatectomía derecha extendida & 1 & 5,8 \\
\hline
\end{tabular}




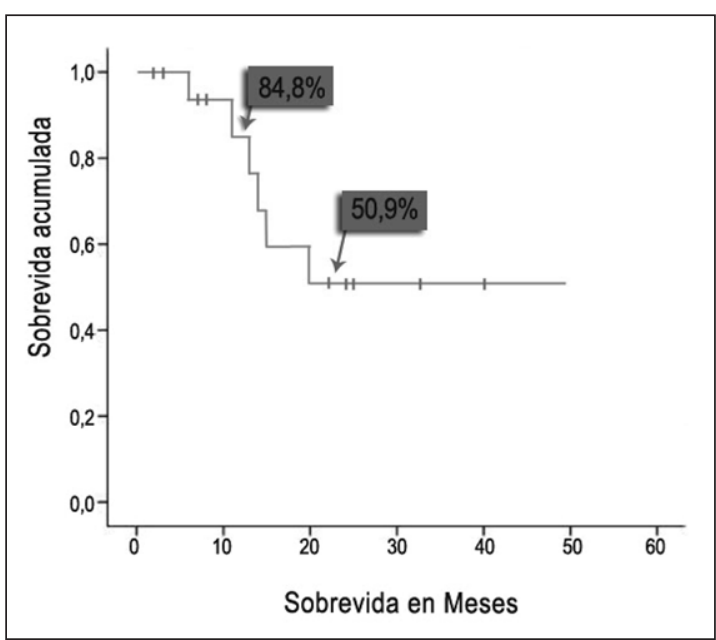

Figura 1. Sobrevida acumulada en pacientes sometidos a resección hepática por metástasis de origen no colorrectal. ellos, portadores de sarcoma, presentaron metástasis hepáticas solamente y los tres pacientes restantes tuvieron metástasis extrahepáticas (el paciente con ameloblastoma y dos pacientes con adenocarcinoma). De los pacientes con recurrencia de metástasis hepática, uno fue sometido a una re-hepatectomía, el cual no presentó complicaciones y ha evolucionado favorablemente, sin registrarse recidiva.

Al realizar análisis univariado de los factores determinantes en la supervivencia de los pacientes sometidos a resección quirúrgica (Tabla 3), se observa que en nuestra serie sólo existe correlación entre la edad y la supervivencia, donde se observa que es menor en los pacientes mayores de 60 años de edad. Los otros factores, en los cuales no se observó correlación, fueron género, momento del diagnóstico de la metástasis, número de metástasis, tamaño tumoral, presencia o no de lesiones tumorales extrahepáticas, tipo de hepatectomía, positividad de los

Tabla 3. Análisis univariado de factores determinantes de sobrevida en pacientes con metástasis hepáticas de origen no colorrectal en quienes se realizó resección hepática tumoral

\begin{tabular}{|c|c|c|c|c|c|}
\hline & Vivo & Muerto & p value & $\mathbf{R R}$ & IC \\
\hline \multicolumn{6}{|l|}{ Edad } \\
\hline$<30$ años & 1 & 0 & 0,04 & 3,64 & $0,61-21,8$ \\
\hline $30-60$ años & 8 & 3 & & & \\
\hline$>60$ años & 1 & 4 & & & \\
\hline \multicolumn{6}{|l|}{ Género } \\
\hline Femenino & 6 & 3 & 0,23 & 1,3 & $0,6-3,07$ \\
\hline Masculino & 4 & 4 & & & \\
\hline \multicolumn{6}{|l|}{ Metástasis } \\
\hline Metacrónica & 8 & 4 & 0,18 & 1,67 & $0,53-5,24$ \\
\hline Sincrónica & 2 & 3 & & & \\
\hline \multicolumn{6}{|l|}{$\mathrm{N}^{\mathrm{o}}$ metástasis } \\
\hline Solitaria & 3 & 5 & 0,18 & 0,48 & $0,18-1,26$ \\
\hline Múltiple & 7 & 2 & & & \\
\hline \multicolumn{6}{|l|}{ Tamaño tumoral } \\
\hline$<5 \mathrm{~cm}$ & 5 & 5 & 0,62 & 0,75 & $0,27-2,06$ \\
\hline$\geq 5 \mathrm{~cm}$ & 2 & 1 & & & \\
\hline \multicolumn{6}{|c|}{ Metástasis extrahepáticas } \\
\hline \multicolumn{6}{|c|}{ Presentes } \\
\hline & 2 & 4 & 0,1 & 0,46 & $0,14-1,5$ \\
\hline & 8 & 3 & & & \\
\hline \multicolumn{6}{|l|}{ Hepatectomía } \\
\hline Limitada & 6 & 4 & 0,47 & 1,05 & $0,46-2,38$ \\
\hline Mayor & 4 & 3 & & & \\
\hline \multicolumn{6}{|l|}{ Márgenes } \\
\hline R0 & 8 & 7 & 0,33 & 0,53 & $0,3-0,86$ \\
\hline $\mathrm{R} 1$ & 1 & 0 & & & \\
\hline \multicolumn{6}{|l|}{ Re-hepatectomía } \\
\hline$(+)$ & 2 & 0 & 0,15 & 1,8 & $1,2-3,01$ \\
\hline$(-)$ & 8 & 7 & & & \\
\hline
\end{tabular}


Tabla 4. Análisis de la sobrevida de los pacientes con metástasis hepáticas según tipo histológico del tumor primario

\begin{tabular}{|c|c|c|c|c|c|}
\hline & Vivo & Muerto & $p$ value & $\mathbf{R R}$ & IC \\
\hline \multicolumn{6}{|c|}{ Sarcoma } \\
\hline Sí & 3 & 3 & 0,58 & 0,79 & $0,31-1,96$ \\
\hline No & 7 & 4 & & & \\
\hline \multicolumn{6}{|c|}{ Adenocarcinoma } \\
\hline Sí & 5 & 3 & 0,77 & 1,13 & $0,51-2,49$ \\
\hline No & 5 & 4 & & & \\
\hline \multicolumn{6}{|c|}{ Estromal } \\
\hline Sí & 0 & 1 & 0,22 & 0 & - \\
\hline No & 10 & 6 & & & \\
\hline \multicolumn{6}{|c|}{ Melanoma } \\
\hline Sí & 1 & 0 & 0,38 & 1,78 & $1,15-2,74$ \\
\hline No & 9 & 7 & & & \\
\hline
\end{tabular}

márgenes quirúrgicos y necesidad o no de realizar re-hepatectomía. Asimismo tampoco hubo diferencia significativa al realizar el análisis comparando la supervivencia según los distintos tipos histológicos de los tumores (Tabla 4).

\section{Discusión}

A la luz de nuestros resultados y de otros similares en la literatura, el tratamiento quirúrgico de los pacientes con metástasis hepáticas de origen no colorrectal es seguro y parece beneficioso en pacientes seleccionados, donde pese a representar una etapa avanzada de la enfermedad tumoral se ha obtenido tasas de supervivencia aceptables y similares a las comunicadas por otros grupos en la literatura ${ }^{10-17,27-30}$. Por lo anterior, en la actualidad, es imperativo evaluar la posibilidad de tratamiento quirúrgico con intención curativa en este grupo de pacientes, así como ya es casi de rutina en los pacientes con metástasis de origen colorrectal, dando opción a aquellos pacientes previamente considerados irresecables, pudiendo entonces ser ofrecido este tratamiento cuando la enfermedad metastásica está bien controlada o responde a la terapia sistémica, posibilitando un beneficio en la supervivencia a largo plazo.

La morbilidad y la mortalidad son factores a considerar cuando se plantea tratamiento quirúrgico agresivo a este tipo de pacientes. En esta serie la morbi-mortalidad es baja a pesar de la gran cantidad de hepatectomías mayores a las que fueron sometidos. Este tipo de intervenciones permite resecar un mayor número de metástasis y de mayor tamaño, con márgenes negativos, sin necesariamente tener mayor impacto en la morbi-mortalidad y sí en términos de supervivencia ${ }^{32-37}$.
A diferencia de otros trabajos con mayor cantidad de pacientes, en nuestro estudio no se observó diferencia significativa entre la supervivencia de los pacientes con distintos tipos de tumor, por lo que es de esperar que al aumentar el número de pacientes sea posible encontrar distintas sobrevidas según histología del tumor primario.

Asociado al tratamiento quirúrgico es crucial el trabajo multidisciplinario en estos pacientes, incluyendo oncólogos médicos y radioterapeutas en la toma de decisiones respecto a la mejor alternativa para cada paciente ${ }^{14,16,17}$. A la vez, es interesante evaluar la utilidad del PET-Scan para pacientes con metástasis con el fin de descartar lesiones extrahepáticas y evitar cirugías complejas en pacientes con enfermedad diseminada.

\section{Referencias}

1. Seymour K, Charnley R. Evidence that metastasis is less common in cirrhotic than normal liver: a systematic review of post-mortem case-control studies. Br J Surg 1999; 86: 1237-1243.

2. Pereira-Lima JE, Lichtenfels E, Barbosa FS, Zetler CG, Kulzynsky JM. Prevalence study of metastases in cirrhotic livers. Hepatogastroenterology 2003; 50: 1490 1495.

3. Martínez J, Jarufe N, González R, Álvarez M. Alternativas terapéuticas actuales de las metástasis hepáticas. Rev Méd Chile 2008; 136: 376-384.

4. Figueras J, González HD . Surgical treatment of breast cancer liver metastasis. The great assignment awaiting spanish hepatic surgery. Cir Esp 2008; 84: 239-240.

5. Robles R, Marín C, Parrilla P. Laparoscopic liver resection for metastatic disease. Minerva Chir 2008; 63: 441-453.

6. Schlitt HJ, Arnold D, Knoefel WT, Ridwelski K, Moehler M, Tannapfel A, et al. Surgical and perioperative 
therapy of liver metastases. Onkologie 2008; 31: 9-13.

7. Fahy BN, D'Angelica M, Dematteo RP, Blumgart LH, Weiser MR, Ostrovnaya I, et al. Synchronous Hepatic Metastases from Colon Cancer: Changing Treatment Strategies and Results of Surgical Intervention. Ann Surg Oncol 2008; 2009; 16: 361-370.

8. Honoré C, Detry O, Deroover A, Piront P, Polus M, Honoré $\mathrm{P}$, et al. When should we resect colorectal liver metastases? Rev Med Liege 2008; 63: 595-599.

9. Taniai N, Akimaru K, Yoshida H, Tajiri T. Surgical treatment for better prognosis of patients with liver metastases from colorrectal cancer. Hepatogastroenterology 2007; 54: 1805-1809.

10. Adam R, Chiche L, Aloia T, Elias D, Salmon R, Rivoire $\mathrm{M}$, et al. Hepatic resection for noncolorectal nonendocrine liver metastases. Analysis of 1452 patients and development of a prognostic model. Ann Surg 2006; 244: 524-535.

11. Detry O, Warzee F, Polus M, De Roover A, Meurisse $\mathrm{M}$, Honoré P. Liver resection for noncolorectal, nonneuroendocrine metastases. Acta Chir Belg 2003; 103 : 458-462.

12. Lindell D, Ohlsson B, Saarela A, Andersson R, Tranberg KG. Liver resection of noncolorectal secondaries. J Surg Oncol 1998; 69: 66-70.

13. Verhoef C, Kuiken BW, Ijzermans JN, de Wilt JH. Partial hepatic resection for liver metastases of noncolorectal origin, is it justified? Hepatogastroenterology 2007; 54: 1517-1521.

14. Choi EA, Abdalla EK. Patient selection and outcome of hepatectomy for noncolorectal non-neuroendocrine liver metastases. Surg Oncol Clin N Am 2007; 16: 557-577.

15. Reddy SK, Barbas AS, Marroquin CE, Morse MA, Kuo PC, Clary BM. Resection of noncolorectal nonneuroendocrine liver metastases: a comparative analisis. J Am Coll Surg 2007; 204: 372-382.

16. Earle SA, Perez EA, Gutiérrez JC, Sleeman D, Livingstone AS, Franceschi D, et al. Hepatectomy enables prolonged survival in select patients with isolated noncolorectal liver metastasis. J Am Coll Surg 2006; 203 : 436-446.

17. Cordera F, Rea DJ, Rodríguez-Davalos M, Hoskin TL, Nagorney DM, Que FG. Hepatic resection for noncolorectal, nonneuroendocrine metastases. J Gastrointest Surg 2005; 9: 1361-1370.

18. Penna C, Nordlinger B. Colorectal metastasis (liver and lung). Surg Clin N Am 2002; 82: 1075-1090.

19. Butte JM, Jarufe N, Bellolio F, Zúñiga A, López F, Pinedo G, y col. Factores pronósticos de pacientes con metástasis hepáticas de cáncer de colon y recto. Rev Chil Cir 2007; 59: 293-298.

20. Dindo D, Demartines N, Clavien PA. Classification of surgical complications: a new proposal with evaluation in a cohort of 6336 patients and results of a survey. Ann Surg 2004; 240: 205-213.

21. Tu R, Xia LP, Yu AL, Wu L. Assessment of hepatic functional reserve by cirrhosis grading and liver volume measurement using CT. World J Gastroenterol 2007; 13 : 3956-3961.

22. Yamanaka J, Saito S, Fujimoto J. Impact of preoperative planning using virtual segmental volumetry on liver resection for hepatocellular carcinoma. World J Surg 2007; 31: 1249-1255.

23. Couinaud C. Surgical anatomy of the liver. Several new aspects. Chirurgie. 1986; 112: 337-342.

24. Couinaud C. The anatomy of the liver. Ann Ital Chir 1992; 63: 693-697.

25. Terminology Committee of the IHPBA. The Brisbane 2000 terminology of hepatic anatomy and resections. HPB 2000; 2: 333-339.

26. Jarufe N, Martínez J, Guzmán S, Pacheco F, Guajardo M, Ibáñez L. Técnica de resecciones hepáticas con disección suprahiliar del pedículo. Rev Chil Cir 2005; 57; 462-469.

27. Shirabe K, Shimada M, Matsumata T, Higashi H, Yakeishi Y, Wakiyama S, et al. Analysis of the prognosis factor for liver metastasis of gastric cancer after hepatic resection: a multi-institutional study of the indications for resection. Hepatogastroenterology 2003; 50: 15601563.

28. Mignon M. Natural history of neuroendocrine enteropancreatic tumors. Digestion 2000; 62: 51-58.

29. Weitz J, Blumgart LH, Fong Y, Jarnagin WR, D'Angelica M, Harrison LE, et al. Parcial hepatectomy for metastases from noncolorectal, nonneuroendocrine carcinoma. Ann Surg 2005; 241: 269-276.

30. Vlastos G, Smith D, Singletary S, Mirza N, Tuttle T, Popat R, et al. Long-term survival after an aggresive surgical approach in patients with breast cancer hepatic metastases. Ann Surg Oncol 2004; 11: 869-874.

31. Yedibela S, Gohl J, Graz V, Pfaffenberger MK, Merkel $\mathrm{S}$, Hohenberger W, Meyer T. Changes in indication and results after resection of hepatic metastases from noncolorectal primary tumors: a single-institutional review. Ann Surg Oncol 2005; 12: 778-785.

32. Guerra JF, Jarufe N, Martínez J, Guzmán S, Pacheco F, Guajardo M y col. Hepatectomía extendida. Una alternativa segura en lesiones hepatobiliares malignas. Rev Chil Cir 2007; 59: 430-435.

33. Karanjia ND, Lordan JT, Quiney N, Fawcett WJ, Worthington TR, Remington J. A comparison of right and extended right hepatectomy with all other hepatic resections for colorectal liver metastases: a ten-year study. Eur J Surg Oncol 2009; 35: 65-70.

34. Abdalla EK, Barnett CC, Doherty D, Curley SA, Vauthey JN. Extended hepatectomy in patients with hepatobiliary malignancies with and without preoperative portal vein embolization. Arch Surg 2002; 137 : 675-680.

35. Vauthey JN, Pawlik TM, Abdalla EK, Arens JF, Nemr $\mathrm{RA}$, Wei SH, et al. Is extended hepatectomy for Hepatobiliary malignancy justified? Ann Surg 2004; 239: $722-732$.

36. Meléndez J, Ferri E, Zwillman M, Fischer M, DeMatteo R, Leung D, et al. Extended hepatic resection: A 6-year retrospective study of risk factors for perioperative mortality. J Am Coll Surg 2001; 192: 47-53.

37. García C, Borda T, Benavides C, Burmeister R, Apablaza S, Rubilar P y cols. Bilirragia y fístula biliar post hepatectomía. Rev Chil Cir 2005; 57: 45-51. 